\title{
The Presence of X-ray Spectral Curvature in HBLs at Different Redshifts
}

\author{
Bidzina Kapanadze ${ }^{* a b c}$, Patrizia Romano ${ }^{b}$, Stefano Vercellone ${ }^{b}$, Sergo Kapanadze \\ ${ }^{a}$, Tengiz Mdzinarishvili ${ }^{a}$, Grigol Kharshiladze ${ }^{d}$ \\ ${ }^{a}$ E. Kharadze Abastumani Astrophysical Observatory, Ilia State University, Colokashvili Av. 3/5, \\ Tbilisi, Georgia, 0162 \\ ${ }^{b}$ INAF, Istituto di Astrofisica Spaziale e Fisica Cosmica, Via U. La Malfa 153, Palermo, Italy, \\ I-90146 \\ ${ }^{c}$ Astronomy Department, University of Michigan, Ann Arbor, MI 48109-1107, USA (guest \\ scientist) \\ ${ }^{d}$ Georgian Technical University, Kostava 77, Tbilisi, Georgia, 0175 \\ E-mail: bidzina_kapanadzedilauni.edu.ge, romanodifc.inaf.it \\ stefano@ifc.inaf.it sergo_kapanadze.1@ilauni.edu.ge \\ tmdzinarishvili@yahoo.com kharshiladze72@mail.ru
}

\begin{abstract}
High-energy peaked BL Lacertae objects (HBLs) mostly show X-ray spectral curvature that provides an effective tool to study the physical conditions and dominant nonstationary processes in HBL jets. Our spectral study confirms the suggestion of some authors that the electrons in the jets of TeV-detected BL Lacertae sources should undergo an effective stochastic acceleration at the shock front yielding a lower curvature compared to the $\mathrm{TeV}$-undetected ones. We have revealed some increase of the curvature parameter with redshift. However, this can also be related to a relatively poor data sampling at larger distances and the further intensive observations of the corresponding sources are necessary.
\end{abstract}

Multifrequency Behaviour of High Energy Cosmic Sources - XI,

25-30 May 2015

Palermo, Italy

\footnotetext{
*Speaker.
} 


\section{Introduction}

BL Lacertae objects (BLLs) are active galactic nuclei (AGN) of elliptical galaxies, which with quasi-featureless spectra, high optical and radio polarization, compact radio-morphology, strong flux variability and broad continuum extending from radio to very high energy $\gamma$-rays. These properties are explained as a result from a relativistically boosted non-thermal emission emanated by the jet closely aligned to the line-of-sight [1]. Their spectral energy distribution (SED) shows the presence of two broad components in the $\log v-\log v F_{v}$ representation. The lower-energy component is explained via the synchrotron radiation emitted by relativistic electrons in the jet, while an inverse Compton (IC) scattering of synchrotron photons by the same electron population is thought to be a source for the high-frequency bump [2].

High-energy peaked BLLs (HBLs) are defined as BLLs with the lower-energy peak situated in the UV/X-ray part of the spectrum [3]. Therefore, these AGNs are expected (a) to be bright in the X-ray band and, therefore, detectable at large distances; (b) to show stronger and faster X-ray flux variability compared to other bands.

Previous X-ray spectral studies of HBLs (e.g. [4] - [11]) have shown that 0.1-10 keV spectra are rather well fitted with the log-parabolic model

$$
F(E)=K\left(E / E_{1}\right)^{-a-b \log \left(E / E_{1}\right)} \mathrm{ph} \mathrm{cm}^{-2} \mathrm{~s}^{-1} \mathrm{keV}^{-1},
$$

( $E$ - photon energy; $K$ - normalization factor; $E_{1}$ - reference energy, generally fixed to $1 \mathrm{keV} ; a$ photon index at $1 \mathrm{keV} ; b$ - curvature parameter) compared to the single powerlaw

$$
F(E)=K E^{-\Gamma},
$$

( $\Gamma$ - the photon index throughout the spectral band). That is, X-ray spectra of HBLs are curved.

Both types of the spectral distribution can be established by systematic acceleration (e.g., electrostatic or electrodynamic) plus some statistical mechanisms [12]. The possible candidates for the log-parabolic case:

- So-called energy-dependent acceleration probability process (EDAP) related to the firstorder Fermi acceleration at the shock wave front moving through the jet. In this case, the emitting particles are confined by a magnetic field with a confinement efficiency decreasing for an increasing gyration radius, and the probability for a further particle acceleration is lower when its energy increases [5].

- Stochastic (e.g. second-order Fermi process developed in the turbulent areas near the shock front) accelerations (obtained via the Fokker-Planck kinetic equation from a mono-energetic electron injection subjected to systematic and stochastic accelerations [13].

The curvature parameter of the log-parabolic energy distribution is inversely proportional to the stochastic acceleration rate [8]. Therefore, synchrotron SED is expected to be relatively broader ( $b \sim 0.3$ ) when the stochastic acceleration is more efficient, while we should observe a narrower SED $(b \sim 0.7)$ in the opposite case [8].

Thus, the presence of X-ray spectral curvature provides an effective tool to investigate the physical conditions and dominant nonstationary processes in HBL jets. The study of spectral curvature in the sources with different redshifts can give an indication about the cosmologic evolution of jet physical properties. 


\section{Results}

Our intensive study [10], [11] of the curvature parameter from the XRT spectra of TeV-emitters 1ES 1959+650 ( $\mathrm{z}=0.048)$ and PKS 2155-304 ( $\mathrm{z}=0.116)$ shows that its distribution has a maximum at $b=0.35-0.37$, i.e. the source mainly exhibited broader synchrotron SEDs, expected when the stochastic mechanism is more efficient ${ }^{1}$. Furthermore, our detection of an anti-correlation between the $b$ parameter and the unabsorbed 0.3-10 keV flux shows a trend of lower curvatures with higher fluxes (i.e. broader SEDs in flaring states) while the higher curvatures are observed mainly during the lower brightness states. This result also favours a stochastic acceleration of the electrons producing X-ray photons during the flares.

However, the latter correlation was the case for the nearest bright HBL Markarian $421(\mathrm{z}=0.031)$ during the BeppoSAX observations performed during 1997-1998 [5]. Our spectral analysis of SwiftXRT observations of 2013 January-May, covering the violent X-ray flaring activities of Mrk 421, show that the $92 \%$ of the spectra are curved with $b=0.04-0.38$ and the distribution maximum at $b$ $=0.22$. The $a$ and $b$ parameters are not correlated, and no significant anti-correlation is found also between $b$ and $F_{0.3-10 \mathrm{keV}}$ - there can be a mix of different acceleration scenarios and "contamination"by cooling processes (A. Tramacere, private communication).

Table 1 presents the summary of preliminary results of the search of X-ray spectral curvature for other HBLs sampled within different radshift ranges. In Column 3, the abbreviation TBL stands for a TeV-detected BLL, and UBL - for the opposite case. Column 5 gives the percentage of the curved spectra with respect the spectra with d.o.f. $>10$. On the basis of these results, we draw some conclusions in the next section.

\section{Conclusions and Summary}

- HBLs show a spectral curvature at any redshift they are detected ( $\mathrm{z}=0.031-0.7)$. However, some sources do not show a curvature from the observations performed do date (e.g., 1ES $0347-121,1 \mathrm{H} 0414+009)$ or the $60-80$ percent of the spectra are described better by the single powerlaw (1ES 2344+514, 1ES 1218+304, Mrk 180, PKS 0548-322, PKS 2005-489, RGB J0710+591, 1ES 0806+524, 1ES 1421+528). This is not simply related to the spectral "poorness". For example, the seven spectra of the brightest HBL source Mrk 421 from the 2013 flaring period are not curved and their degrees of freedom are $63-305$.

- In average, HBLs show an increasing curvature with redshift but this result can be an artifact of a poor data sampling at larger $\mathrm{z}$ values. In that case, there are much fewer observations and lower fluxes yielding relatively poor spectra. It is almost impossible to detect spectral curvature for the spectra with d.o.f. $<10$ (that corresponds to $t_{\mathrm{obs}}<3 \mathrm{ks}$ for the Swift-XRT count rate of about $0.3 \mathrm{cts} \mathrm{s}^{-1}$ ). It is not a good idea to add short observations to obtain a richer spectrum - BLLs show large and fast spectral variability and a sum of the spectra of different curvature can lead to an unacceptable value of reduced Chi-square.

\footnotetext{
${ }^{1}$ We have used HeAsoft versions 6.15 - 6.16 and Swift-XRT CALDB versions 20131223 - 20140120 to process the raw X-ray data.
} 
Table 1: Results of the search of X-ray spectral curvature in HBLs.

\begin{tabular}{|c|c|c|c|c|c|}
\hline $\begin{array}{l}\text { Source } \\
\text { (1) }\end{array}$ & $\begin{array}{c}\mathrm{z} \\
(2)\end{array}$ & $\begin{array}{c}\text { TBL/UBL } \\
\text { (3) }\end{array}$ & $\begin{array}{l}b \\
(4)\end{array}$ & $\begin{array}{l}\% \\
(5)\end{array}$ & $\begin{array}{l}\text { Citation } \\
\text { (6) }\end{array}$ \\
\hline \multicolumn{6}{|c|}{$z \leq 0.05$} \\
\hline Mrk 501 & 0.034 & TBL & $0.08-0.42^{a}$ & 85 & TW \\
\hline RX J0214.2+5144 & 0.049 & UBL & $0.41-0.65$ & 100 & [8] \\
\hline Mrk 180 & 0.046 & TBL & $0.17-0.74$ & 39 & This work \\
\hline 1ES $2344+514$ & 0.046 & TBL & $0.17-0.74$ & 39 & This work \\
\hline \multicolumn{6}{|c|}{$0.05<z \leq 0.1$} \\
\hline 1ES $1727+502$ & 0.055 & TBL & $0.28-0.65$ & 62 & This work \\
\hline 1RXS J001356.6-18540 & 0.094 & TBL & $0.36-0.68$ & 100 & [8] \\
\hline PKS 0548-322 & 0.069 & TBL & $0.08-0.52$ & 47 & This work, [7] \\
\hline 1ES $1741+196$ & 0.080 & TBL & $0.35-0.47$ & 67 & [8] \\
\hline PKS 2005-489 & 0.071 & TBL & $-0.23-0.70$ & 35 & This work, [7] \\
\hline \multicolumn{6}{|c|}{$0.1<z \leq 0.15$} \\
\hline H $1426+428$ & 0.129 & TBL & -0.22 Ü 0.28 & 54 & This work \\
\hline 1ES $0323+022$ & 0.147 & UBL & $0.42-0.43$ & 100 & [8] \\
\hline RGB J0710+591 & 0.125 & TBL & $0.17-0.23$ & 21 & This work \\
\hline $1 \mathrm{ES} 0806+524$ & 0.138 & TBL & $0.28-0.33^{b}$ & 20 & This work \\
\hline 1RXS J101015.9-31190 & 0.143 & UBL & $0.46-0.76$ & 100 & [8] \\
\hline RGB J1053+494 & 0.140 & UBL & $1.01(0.46)$ & 100 & [8] \\
\hline RX J1136.5+6737 & 0.136 & TBL & $0.38-0.96$ & 100 & This work, $[8]$ \\
\hline 1ES $1255+244$ & 0.141 & UBL & $0.55(0.28)$ & 100 & [8] \\
\hline 1RXS J151040.8+33351 & 0.114 & UBL & $0.45(0.11)$ & 100 & [8] \\
\hline \multicolumn{6}{|c|}{$0.15<z \leq 0.2$} \\
\hline 1ES $1218+304$ & 0.182 & TBL & $0.15-0.37$ & 33 & This work \\
\hline 1RXS J075124.3+17304 & 0.185 & UBL & $1.33(0.88)$ & 100 & [8] \\
\hline RGB J0916+526 & 0.190 & UBL & $0.55(0.18)$ & 100 & [8] \\
\hline 1RXS J093037.1+49502 & 0.187 & UBL & $0.89(0.15)$ & 100 & [8] \\
\hline 1RXS J095225.8+75021 & 0.179 & UBL & $0.44-0.58$ & 100 & [8] \\
\hline 1ES $1101-232$ & 0.186 & TBL & $0.14-0.78$ & 71 & This work, [7] \\
\hline 1RXS J114535.8-03394 & 0.167 & UBL & $1.02-1.26$ & 100 & [8] \\
\hline BZB J1231+6414 & 0.163 & UBL & $0.25(0.08)$ & 100 & [8] \\
\hline 1RXS J125341.2-39320 & 0.179 & UBL & $0.41(0.21)$ & 100 & [8] \\
\hline RGB J1341+399 & 0.172 & UBL & $0.50-0.70$ & 86 & [8] \\
\hline 1ES $1440+122$ & 0.163 & TBL & $0.32-1.36$ & 80 & [8] \\
\hline 1RXS J220156.0-17065 & 0.169 & UBL & $0.40-0.92$ & 67 & [8] \\
\hline Н 2356-309 & 0.165 & TBL & $0.22-0.78$ & 100 & This work, [7] \\
\hline \multicolumn{6}{|c|}{$0.2<z \leq 0.3$} \\
\hline 1ES 1011+496 & 0.212 & TBL & $0.18-0.39$ & 56 & This work \\
\hline 1ES $0120+340$ & 0.272 & UBL & $0.25-0.35$ & 11 & This work \\
\hline 1ES $0158+003$ & 0.298 & UBL & $0.69(0.28)$ & 100 & [8] \\
\hline 1RXS J105607.0+02521 & 0.236 & UBL & $0.59(0.15)$ & 100 & [8] \\
\hline 1RXS J123739.2+62584 & 0.297 & UBL & $0.44-1.02$ & 66 & [8] \\
\hline 1RXS J141756.8+25432 & 0.237 & UBL & $0.43-0.88$ & 86 & [8] \\
\hline 1RXS J160518.5+54210 & 0.212 & UBL & $0.84(0.33)$ & 100 & [8] \\
\hline \multicolumn{6}{|c|}{$0.3<z \leq 0.4$} \\
\hline 1ES $0502+675$ & 0.314 & TBL & $0.28-0.70$ & 100 & This work \\
\hline 1RXS J020837.5+35231 & 0.318 & UBL & $0.41-0.61$ & 100 & {$[8]$} \\
\hline EXO 0556.4-3838 & 0.302 & UBL & $0.28-0.74$ & 100 & This work \\
\hline 1ES $0737+746$ & 0.314 & UBL & $0.16-0.18$ & 100 & [8] \\
\hline 1RXS J143917.7+39324 & 0.341 & UBL & $0.64(0.29)$ & 100 & [8] \\
\hline 1RXS J234332.5+34395 & 0.366 & UBL & $0.82-1.34$ & 100 & [8] \\
\hline
\end{tabular}

${ }^{a}$ From the prolonged flaring activity during 2014 April-October observed with Swift-XRT; no significant correlation between the $\mathrm{a}$ and $\mathrm{b}$ as well between $\mathrm{b}$ and $\mathrm{F} 0.3-10 \mathrm{keV}$ is found.

${ }^{b}$ marginal detection. 
Table 1: - Continued.

\begin{tabular}{cccccc}
\hline Source & $\mathrm{z}$ & TBL/UBL & $b$ & $\%$ & Citation \\
\hline PG 1553+113 & $0.4-0.58$ & TBL & $0.17-0.39^{a}$ & 72 & This work \\
1RXS J022716.6+02015 & 0.456 & UBL & $0.49-0.70$ & 100 & {$[8]$} \\
1RXS J044229.8-00182 & 0.449 & UBL & $0.45(0.32)$ & 100 & {$[8]$} \\
1RXS J062150.0-34114 & 0.529 & UBL & $0.90(0.52)$ & 100 & {$[8]$} \\
1RXS J213135.5-09152 & 0.449 & UBL & $0.76(0.22)$ & 100 & {$[8]$} \\
\hline & 0.7 & Z $>0.6$ & & & \\
\hline 1H 1515+660 & 0.610 & UBL & $0.18-0.53$ & 80 & This work \\
1RXS J003334.6-192130 & 0.617 & UBL & $0.47(0.15)$ & 33 & This work \\
1RXS J020413.6-333345 & 0.672 & UBL & $0.37-0.87$ & 25 & This work \\
1RXS J083251.9+330011 & 0.601 & UBL & $0.58-1.06$ & 60 & This work work \\
1RXS J113755.4-171034 & 0.638 & UBL & $0.37-0.59$ & 40 & This work \\
1ES 1421+582 & $0.89(?)$ & UBL & $0.26-0.89$ & 100 & This work \\
1ES 1533+535 & & & & &
\end{tabular}

${ }^{a}$ From two flares observed during the Swift-XRT 2012 and 2014 campaigns, respectively.

- Our results are mainly in agreement with the prediction of [8] that the electrons in the TBL jets should undergo a more efficient stochastic acceleration than in the UBL jets - they mostly show lower curvature (三 wider SEDs) to be established in this case.

- TeV emission can be expected from following UBLs: 1ES 0120+340, EXO 0556.4-3838, 1ES $0737+746$, BZB J1231+6414, 1H 1515+660 - due to the presence of a low curvature. The extragalacric background light restricts their TeV-detection but they are closer than the farthest $\mathrm{TeV}$ source S3 0218+35 ( $\mathrm{z}=0.944)$. Although other UBLs show higher curvatures that makes them unsuitable candidates for a TeV-detection, intensive study of nearby HBLs show that they sometimes also show high curvatures (above the "dividing line" $b=0.55$, derived in [8]), and they show large spectral variability with $b=0.3-0.4$ that can be also the case for UBLs, leading to smaller curvatures in some epochs. Nevertheless, there are a few observations of UBLs allowing to reveal spectral curvature and, in many cases, the $b$ parameter is determined below the $3 \sigma$ significance and its high values can be simply related to larger errors. Therefore, more observations are needful to draw more firm conclusions.

\section{Acknowledgements}

B.K. thanks Dr. Maya Todua, the director of E. Kharadze Abastumani Astrophysical Observatory, and Prof. David Tarkhnishvili, the dean of the Faculty of Natural Sciences and Engineering at Ilia State University (Tbilisi, Georgia), for a financial support to participate in the conference. P.R. acknowledges the contract ASI-INAFI/004/11/0. This research has made use of XRTDAS. We acknowledge the helpful service of HEASARC.

\section{References}

[1] M. C. Urry \& P. Padovani, Unified Schemes for Radio-Loud Active Galactic Nuclei, PASP, 107, 30 (803)

[2] A. Celotti \& G. Ghisellini, The power of blazar jets, MNRAS, 385, 283 (2008) 
[3] P. Padovani \& P. Giommi, The Connection Between X-Ray-And Radio-Selected Bl Lacertae Objects, ApJ, 444, 567 (1995)

[4] E. S. Perlman et al., Intrinsic Curvature in The X-Ray Spectra Of Bl Lacertae Objects, ApJ, 625, 727 (2005)

[5] E. Massaro et al., Log-parabolic spectra and particle acceleration in the BL Lac object Mkn 421: Spectral analysis of the complete BeppoSAX wide band X-ray data set, A\&A, 413,489 (2004)

[6] E. Massaro et al., Log-parabolic spectra and particle acceleration in blazars III. SSC emission in the TeV band from Mkn 501, A\&A, 448, 861 (2006)

[7] F. Massaro et al., X-ray spectral evolution of TeV BL Lacertae objects: eleven years of observations with BeppoSAX, XMM-Newton and Swift satellites, A\&A, 478, 395 (2008)

[8] F. Massaro et al., X-Ray and Tev Emissions From High-Frequency-Peaked Bl Lac Objects, ApJ, 742, L32 (2011)

[9] A. Tramacere et al., Swift observations of the very intense flaring activity of Mrk 421 during 2006. I. Phenomenological picture of electron acceleration and predictions for MeV/GeV emission, A\&A, 501, 879 (2009)

[10] B. Kapanadze et al., The X-ray behaviour of the high-energy peaked BL Lacertae source PKS 2155-304 in the 0.3-10 keV band, MNRAS, 444,1076 (2014)

[11] B. Kapanadze et al., The long-term Swift observations of high-energy peaked BL Lacertae source IES $1959+650$, in preparation, submitted to MNRAS

[12] A. Tramacere et al., Stochastic Acceleration and the Evolution of Spectral Distributions in Synchro-Self-Compton Sources: A Self-Consistent Modeling Of BlazarsŠ Flares, ApJ, 739, 66 (2011)

[13] A. Paggi et al., SSC radiation in BL Lacertae sources, the end of the tether, A\&A, 504, 821 (2009)

\section{DISCUSSION}

JIM HOWARTH BEALL: Is it possible to distinguish among the radiations coming from accretion disc and jet, respectively?

BIDZINA KAPANADZE: There is a clear difference between the spectra corresponding to jet and accretion disc. In the latter case, we observe prominent emission lines while the spectra are featureless for the radiation emanated by a jet that is the case for X-ray spectra of BL Lacertae objects, in contrast to flat-spectrum radio quasars (FSRQs) and Seyfert galaxies.

WOLFGANG KUNDT: Present-day astrophysics is full of erroneous 'conclusions', because it lacks (stabilizing) tests. Stochastic particle acceleration to high energies is one of them; it easily violates the law of non-decreasing entropy.

BIDZINA KAPANADZE: The electron acceleration to highest energies in BL Lac jets is not due to only stochastic acceleration. Namely, there should be a combination of systematic coherent acceleration, responsible for the energy peak position of the log-parabolic spectrum, and of stochastic acceleration, which accounts for the SED broadening around its peak related to the lower spectral curvature (see, e. g., [8]). 